\title{
HOMOTOPY-DETERMINANT ALGORITHM FOR SOLVING NONSYMMETRIC EIGENVALUE PROBLEMS
}

\author{
T. Y. LI AND ZHONGGANG ZENG
}

\begin{abstract}
The eigenvalues of a matrix $A$ are the zeros of its characteristic polynomial

$$
f(\lambda)=\operatorname{det}[A-\lambda I] .
$$

With Hyman's method of determinant evaluation, a new homotopy continuation method, homotopy-determinant method, is developed in this paper for finding all eigenvalues of a real upper Hessenberg matrix. In contrast to other homotopy continuation methods, the homotopy-determinant method calculates eigenvalues without computing their corresponding eigenvectors. Like all homotopy methods, our method solves the eigenvalue problem by following eigenvalue paths of a real homotopy whose regularity is established to the extent necessary. The inevitable bifurcation and possible path jumping are handled by effective processes.

The numerical results of our algorithm, and a comparison with its counterpart, subroutine HQR in EISPACK, are presented for upper Hessenberg matrices of numerous dimensions, with randomly generated entries. Although the main advantage of our method lies in its natural parallelism, the numerical results show our algorithm to be strongly competitive also in serial mode.
\end{abstract}

\section{INTRODUCTION}

Consider the eigenvalue problem

$$
A \mathbf{x}=\lambda \mathbf{x},
$$

where $A$ is an $n \times n$ nonsymmetric matrix and $\mathbf{x}=\left(x_{1}, \ldots, x_{n}\right)^{\top}$. By an orthogonal transformation, $A$ is similar to a matrix in upper Hessenberg form. Hence we shall assume throughout this paper that $A$ is an upper Hessenberg matrix,

$$
A=\left(a_{i j}\right)=\left(\begin{array}{cccccc}
* & * & * & \ldots & \ldots & * \\
a_{21} & * & * & \ldots & \ldots & * \\
& a_{32} & * & \ldots & \ldots & * \\
& & \ldots & \ldots & \ldots & \ldots \\
& 0 & & a_{n-1, n-2} & * & * \\
& & & & a_{n, n-1} & *
\end{array}\right) .
$$

Received by the editor February 25, 1991 and, in revised form, December 2, 1991 and January 29, 1992.

1991 Mathematics Subject Classification. Primary 65F15, 65F40, 65H05, 65H17, 58C99, 58F14.

This research was supported in part by NSF Grant CCR-9024840. 
We further assume $A$ is irreducible, that is, none of the subdiagonal entries $a_{j, j-1}, j=2, \ldots, n$, are zero; otherwise, we can consider a reduced matrix.

The purpose of this paper is to use the homotopy continuation method to find all eigenvalues of $A$. Homotopy continuation methods have been successfully applied to symmetric tridiagonal eigenvalue problems with remarkable numerical results $[10,14]$. The implementation of the continuation algorithm for the real nonsymmetric case has been discussed in detail in [13]. While the algorithm in [13] calculates the eigenvalue and its corresponding eigenvector simultaneously, the method we propose here focuses on finding only the eigenvalues of $A$ without computing the eigenvectors. Our homotopy deforms the characteristic equation of $A$ into the characteristic equation of a matrix $D$ with known eigenvalues. To distinguish our homotopy from the previous one in [13], we shall name our method the homotopy-determinant algorithm.

For $t \in[0,1]$, let

$$
A(t)=\left(a_{i j}(t)\right)=(1-t) D+t A,
$$

where $D=\left(d_{i j}\right)$ is a matrix, usually called the initial matrix, also in upper Hessenberg form but with known eigenvalues. Let $H: \mathbf{C} \times[0,1] \rightarrow \mathbf{C}$ be defined by

$$
H(\lambda, t)=\operatorname{det}[A(t)-\lambda I] .
$$

Let $H_{\lambda}$ and $H_{t}$ denote the partial derivatives of $H$ with respect to $\lambda$ and $t$, respectively. When $\mathscr{D} H=\left(H_{\lambda}, H_{t}\right)$ is of full rank at $\left(\lambda^{0}, t^{0}\right) \in H^{-1}(0)$, then locally the solution set of $H(\lambda, t)=0$ consists of a smooth 1-manifold $(\lambda(s), t(s))$ passing through $\left(\lambda^{0}, t^{0}\right)$. We shall call such a curve $(\lambda(s), t(s))$ an eigenvalue path. These eigenvalue paths connect the eigenvalues of $D$ and those of $A$ and satisfy the ordinary differential equation

$$
H_{\lambda} \frac{d \lambda}{d s}+H_{t} \frac{d t}{d s}=0 .
$$

To effectively follow the eigenvalue paths for finding all eigenvalues of $A$, one must efficiently evaluate $H, H_{\lambda}$, and $H_{t}$. For this purpose, the method of Hyman [7, 18] for evaluating determinants of Hessenberg matrices and their derivatives will be used. The details will be discussed in $\S 2$.

The regularity of the eigenvalue paths needed for a general homotopy algorithm is usually achieved by random perturbation of certain parameters. To maintain the conjugacy of the eigenvalues of $A(t)$ for each $t$, so that when a complex eigenvalue path $(\lambda(s), t(s))$ is followed its conjugate eigenvalue path is obtained as a by-product without further computations, we restrict the perturbation to be real. In contrast to the complex perturbation used in $[2,3,14$, 12], the real perturbation cannot maintain complete regularity of our eigenvalue paths. Indeed, bifurcations on some of the eigenvalue paths are inevitable. In $\S 3$, we shall establish the regularity of our eigenvalue paths to an extent necessary and analyze the bifurcation behavior. It turns out that the necessary regularity can be obtained by the perturbation of only four entries of the initial matrix, regardless of the size of the matrix $A$.

It is desirable to choose $D$ as close to $A$ as possible, so that the eigenvalue paths are close to straight lines and thus easy to follow. So, we employ the strategy of "divide and conquer." The initial matrix $D=\left(d_{i j}\right)$ is formed by 
making one of the subdiagonal entries $a_{p+1, p}$ of $A$ zero, namely,

$$
d_{i j}= \begin{cases}a_{i j}, & (i, j) \neq(p+1, p), \\ 0, & (i, j)=(p+1, p),\end{cases}
$$

and the eigenvalues of $D$ are obtained by solving the eigenvalues of the reduced submatrices of $D$. We conquer the matrix $A$ by following the eigenvalue paths of our homotopy in (1.2). The algorithm of following the eigenvalue path will be described in detail in $\S 4$.

The numerical results of our algorithm on upper Hessenberg matrices with randomly generated entries are presented in $\S 5$. The results seem very encouraging. The well-developed $\mathrm{QR}$ algorithm for nonsymmetric eigenvalue problems implemented in EISPACK [16], the subroutine HQR, is widely considered to be the most efficient algorithm available. Compared with HQR, our algorithm is strongly competitive in terms of both accuracy and speed on the examples we have tried.

Scientific and engineering research is becoming increasingly dependent upon the development and implementation of efficient parallel algorithms on modern high-performance computers. The search for methods of solving eigenvalue problems on advanced computers has produced several algorithms, such as Divide and Conquer [4, 6] and Bisection/Multisections [8, 9, 15], for symmetric tridiagonal matrices. Good parallel algorithms for nonsymmetric eigenvalue problems, however, are still in demand. The most important feature of our algorithm is its natural parallelism, in the sense that each eigenvalue path is traced independently of the others. In this respect, it stands in contrast to the highly serial QR algorithm. The parallel implementation using $n$ processors should increase the efficiency of our algorithm by a full power of $n$, making it an excellent candidate for advanced computer architectures. Reports on this important aspect will be given in a separate paper.

Concurrent and independent research on parallel computation for nonsymmetric eigenvalue problems has been carried out by Dongarra and Sidani [5]. Their approach also involves the strategy of "divide and conquer." In contrast to our algorithm, their method must compute the eigenvalues and associated eigenvectors at the same time.

\section{HYMAN'S METHOD}

For $A(t)=\left(a_{i j}(t)\right)=(1-t) D+t A$ and $\mathbf{x}=\left(x_{1}, \ldots, x_{n}\right)^{\top}$, the system of equations $(A(t)-\lambda I) \mathbf{x}=0$ can be written as

$$
\begin{aligned}
& \left(a_{11}(t)-\lambda\right) x_{1}+a_{12}(t) x_{2}+\cdots+a_{1 n}(t) x_{n}=0 \\
& a_{21}(t) x_{1}+\left(a_{22}(t)-\lambda\right) x_{2}+\cdots+a_{2 n}(t) x_{n}=0 \\
& a_{k, k-1}(t) x_{k-1}+\left(a_{k k}(t)-\lambda\right) x_{k}+\cdots+a_{k n}(t) x_{n}=0 \\
& a_{n, n-1}(t) x_{n-1}+\left(a_{n n}(t)-\lambda\right) x_{n}=0 .
\end{aligned}
$$

Given a value of $\lambda$, and setting $x_{n}=1$ in the last equation, we can solve the last $n-1$ equations recursively for $x_{n-1}, \ldots, x_{2}, x_{1}$. These values are then used to evaluate the left-hand side of the first equation,

$$
F(\lambda, t)=\left(a_{11}(t)-\lambda\right) x_{1}+a_{12}(t) x_{2}+\cdots+a_{1 n}(t) x_{n} .
$$


Obviously, $F(\lambda, t)=0$ precisely if $\lambda$ is an eigenvalue of $A(t)$. The matrix $A(t)-\lambda I$ has the form

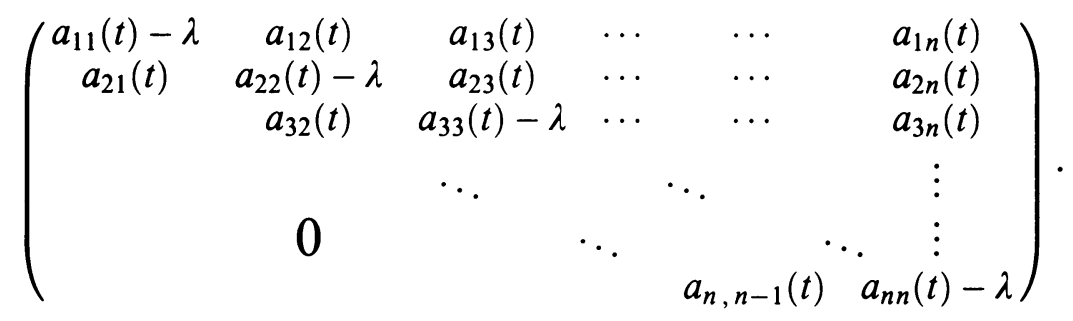

For $j=1,2, \ldots, n$ we multiply the $j$ th column by $x_{j}$ as found above and add this to the last column, thereby obtaining the matrix

$$
\left(\begin{array}{ccccccc}
a_{11}(t)-\lambda & a_{12}(t) & a_{13}(t) & \cdots & \cdots & a_{1, n-1}(t) & F(\lambda, t) \\
a_{21}(t) & a_{22}(t)-\lambda & a_{23}(t) & \cdots & \cdots & a_{2, n-1}(t) & 0 \\
& a_{32}(t) & a_{33}(t)-\lambda & \cdots & \cdots & a_{3, n-1}(t) & 0 \\
& & \ddots & & & \vdots & \vdots \\
& 0 & & \ddots & & \vdots & \vdots \\
& & & & & a_{n, n-1}(t) & 0
\end{array}\right) .
$$

We then have

$$
H(\lambda, t)=\operatorname{det}[A(t)-\lambda I]=(-1)^{n-1} F(\lambda, t) \prod_{j=1}^{n-1} a_{j+1, j}(t)
$$

To compute $H_{\lambda}$, we need to compute $\frac{\partial F}{\partial \lambda}$. Differentiating (2.1) with respect to $\lambda$, taking into account that the $x_{j}, j=1, \ldots, n-1$, are functions of $(\lambda, t)$, and $x_{n}=1$, yields

$$
\begin{aligned}
& \left(a_{11}(t)-\lambda\right) \frac{\partial x_{1}}{\partial \lambda}-x_{1}+a_{12}(t) \frac{\partial x_{2}}{\partial \lambda}+\cdots+a_{1 n}(t) \frac{\partial x_{n}}{\partial \lambda}=0 \\
& a_{21}(t) \frac{\partial x_{1}}{\partial \lambda}+\left(a_{22}(t)-\lambda\right) \frac{\partial x_{2}}{\partial \lambda}-x_{2}+\cdots+a_{2 n}(t) \frac{\partial x_{n}}{\partial \lambda}=0 \\
& a_{k, k-1}(t) \frac{\partial x_{k-1}}{\partial \lambda}+\left(a_{k k}(t)-\lambda\right) \frac{\partial x_{k}}{\partial \lambda}-x_{k}+\cdots+a_{k n}(t) \frac{\partial x_{n}}{\partial \lambda}=0 \\
& a_{n, n-1}(t) \frac{\partial x_{n-1}}{\partial \lambda}+\left(a_{n n}(t)-\lambda\right) \frac{\partial x_{n}}{\partial \lambda}-x_{n}=0 .
\end{aligned}
$$

With $\partial x_{n} / \partial \lambda=0$, we solve (2.3) successively for $\partial x_{n-1} / \partial \lambda, \ldots, \partial x_{1} / \partial \lambda$, using the previously computed values of $x_{1}, \ldots, x_{n}$. The value of the lefthand side of the first equation in (2.3) is then $\frac{\partial F}{\partial \lambda}$. We proceed similarly for $H_{t}$.

The method described above, basically Gaussian elimination without pivoting, is due to Hyman [7, 18]. The backward stability of this method was established by Wilkinson in [18]. It was proved that there is little correlation between the accuracy of the method and the magnitude of the subdiagonal entries of $A(t)$, although it may seem that some subdiagonal entries with small magnitude would endanger the accuracy of the procedure. 


\section{REGULARITY AND BIFURCATION}

The boundedness as well as the smoothness of the eigenvalue paths are the essential properties needed for the homotopy method. In our case, the eigenvalue paths are always bounded in $\mathbf{C} \times[0,1]$ because of the continuity of the eigenvalue with respect to the entries of the matrix. The smoothness of the homotopy paths can usually be achieved by random perturbation of certain parameters. To maintain our homotopy real for important practical considerations, we restrict the perturbation to real perturbation. In contrast to complex perturbations used in $[2,3,11,12]$, real perturbations cannot guarantee the smoothness of our eigenvalue paths, and bifurcations, which do not occur when complex perturbations are used, are inevitable. In [13], regularity and bifurcation results were obtained by using real perturbations on all the upper triangular entries. In our case, we will show that perturbation of as few as four entries in the initial matrix is sufficient to achieve the smoothness of the eigenvalue paths to the extent necessary, along with the simplicity of the bifurcation behavior.

For the initial matrix $D=\left(d_{i j}\right)$ in upper Hessenberg form, with one of the subdiagonal entries $d_{p+1, p}=0$ and $d_{j+1, j} \neq 0$ for $j \neq p$, we shall take $d_{1 p}, d_{1, n-1}, d_{1 n}$, and $d_{p+1, n}$ as the only parameters subject to perturbation. We write

$$
\begin{aligned}
& D=\left(\begin{array}{cccccccc}
d_{11} & \cdots & \cdots & \mathbf{d}_{1 p} & d_{1, p+1} & \cdots & \mathbf{d}_{1, n-1} & \mathbf{d}_{1 n} \\
d_{21} & \ddots & & \vdots & \vdots & & \vdots & \vdots \\
& \ddots & \ddots & \vdots & \vdots & & \vdots & \vdots \\
& & d_{p, p-1} & d_{p p} & d_{p, p+1} & \cdots & d_{p, n-1} & d_{p n} \\
& & & 0 & d_{p+1, p+1} & \cdots & \cdots & \mathbf{d}_{p+1, n} \\
& & & & d_{p+2, p+1} & \ddots & & \vdots \\
& & & & & \ddots & \ddots & \vdots \\
& & & & & & d_{n, n-1} & d_{n n}
\end{array}\right) \\
& =\left(\begin{array}{cc}
D_{1} & * \\
0 & D_{2}
\end{array}\right),
\end{aligned}
$$

where

$$
\begin{aligned}
D_{1} & =\left(\begin{array}{cccc}
d_{11} & \cdots & \cdots & \mathbf{d}_{1 p} \\
d_{21} & \ddots & & \vdots \\
& \ddots & \ddots & \vdots \\
0 & & d_{p, p-1} & d_{p p}
\end{array}\right), \\
D_{2} & =\left(\begin{array}{cccc}
d_{p+1, p+1} & \cdots & \cdots & \mathbf{d}_{p+1, n} \\
d_{p+2, p+1} & \ddots & & \vdots \\
0 & \ddots & \ddots & \vdots \\
0 & & d_{n, n-1} & d_{n n}
\end{array}\right) .
\end{aligned}
$$

Proposition 3.1. For the homotopy $H(\lambda, t)=\operatorname{det}[A(t)-\lambda I]$ with $A(t)=$ $(1-t) D+t A$ there exists a subset $Q$ in $\mathbf{R}^{4}$ with full measure, i.e., $\mathbf{R}^{4} \backslash Q$ has 


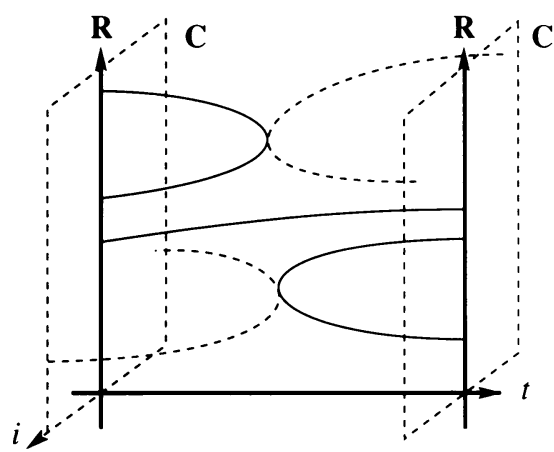

Paths in $\mathbf{C} \times[0,1]$

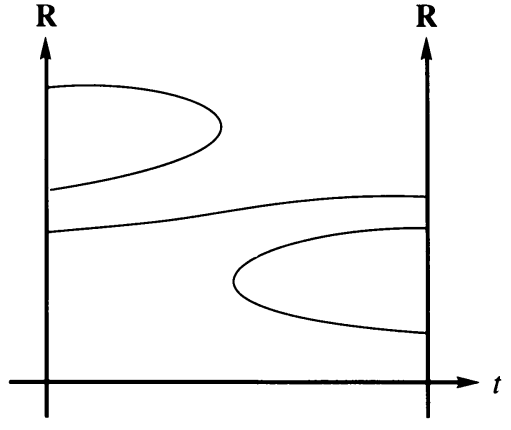

Paths restricted in $\mathbf{R} \times[0,1]$

FIGURE 1. Smoothness of real eigenvalue paths

zero Lebesgue measure, such that if $\left(d_{1 p}, d_{p+1, n}, d_{1, n-1}, d_{1 n}\right)$ is in $Q$, then

(i) the initial matrix $D$ has no multiple eigenvalues;

(ii) for $(\lambda, t) \in H^{-1}(0)$ with complex $\lambda, H_{\lambda} \neq 0$ holds;

(iii) if $H$ is considered a map from $\mathbf{R} \times[0,1) \rightarrow \mathbf{R}$, then zero is a regular value of $H$, i.e., for every $(\lambda, t) \in H^{-1}(0)$ with real $\lambda,\left(H_{\lambda}, H_{t}\right)$ is of rank 1 .

From (iii) above, by the Implicit Function Theorem, there exists a real eigenvalue path passing through any $(\lambda, t) \in H^{-1}(0)$ with real $\lambda$. By a standard continuation argument, if $H$ is considered a map from $\mathbf{R} \times[0,1) \rightarrow \mathbf{R}$, then $H^{-1}(0)$ consists of real one-dimensional manifolds, and no bifurcation occurs in $\mathbf{R} \times[0,1)$, as shown in Figure 1 .

In $\mathbf{C} \times[0,1)$, from (ii), the Implicit Function Theorem also guarantees the regularity of the local eigenvalue path at $(\lambda, t) \in H^{-1}(0)$ with complex $\lambda$. Therefore, in $\mathbf{C} \times[0,1)$, bifurcation can only occur at a real point $\left(\lambda^{0}, t^{0}\right) \in$ $H^{-1}(0)$ at which $H_{\lambda}$ must vanish, i.e., $\lambda^{0}$ is a multiple eigenvalue of $A\left(t^{0}\right)$. Otherwise, $\mathscr{D} H=\left(H_{\lambda}, H_{t}\right)$ at $\left(\lambda^{0}, t^{0}\right)$, considered a real $2 \times 3$ matrix, has the form

$$
\left(\begin{array}{ccc}
a & 0 & * \\
0 & a & *
\end{array}\right)
$$

with $a \equiv H_{\lambda} \neq 0$, which is of rank 2 (full rank).

In summary, at a bifurcation point $\left(\lambda^{0}, t^{0}\right)$, since $\lambda^{0}$ must be real and a regular point in $\mathbf{R} \times(0,1)$, there is a real eigenvalue path passing through it. But no other real eigenvalue path can pass through $\left(\lambda^{0}, t^{0}\right)$, since bifurcation does not occur in $\mathbf{R} \times[0,1)$. Hence, other bifurcation branches must consist of complex eigenvalue paths.

It is easy to see that the number of bifurcation branches at a bifurcation point $\left(\lambda^{0}, t^{0}\right)$ equals the multiplicity of $\lambda^{0}$ as an eigenvalue of $A\left(t^{0}\right)$, because of the continuity of eigenvalues with respect to $t$ and the fixed number of eigenvalues of $A(t)$, counting multiplicities. We will show in Proposition 3.6 that generically the multiplicity of $\lambda^{0}$ is no more than two. Therefore, there are only two bifurcation branches at $\left(\lambda^{0}, t^{0}\right)$, consisting of one complex eigenvalue path and one real eigenvalue path, as shown in Figure 2. 

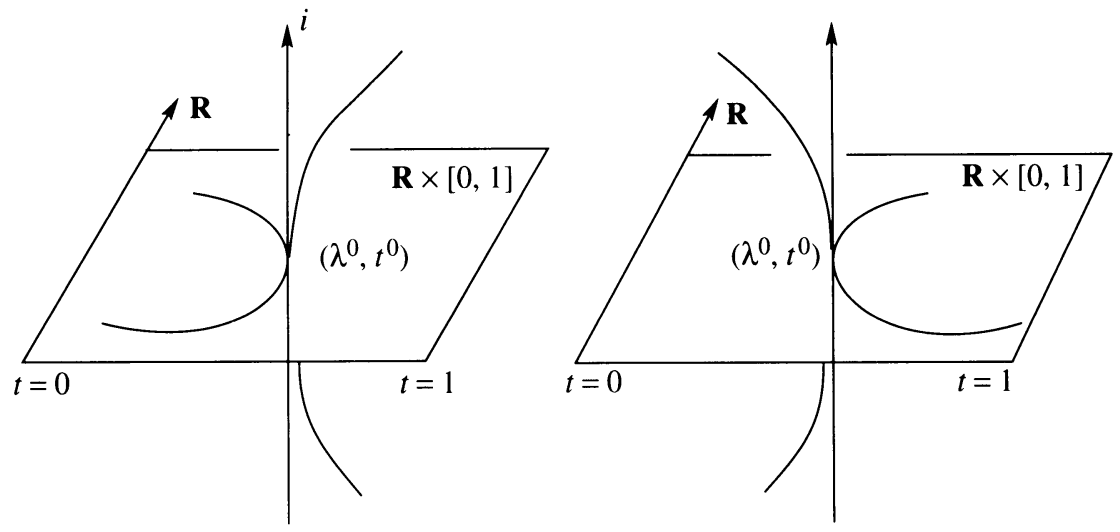

FIGURE 2. Bifurcation behavior

Notice that $\dot{t}\left(=\frac{d t}{d s}\right)$ must vanish at $\left(\lambda^{0}, t^{0}\right)$, since $H_{\lambda}=0, H_{t} \neq 0$, and $H_{\lambda} \dot{\lambda}+H_{t} \dot{t}=0$. When arc length is used as the parameter of the path $(\lambda(s), t(s))$, the tangent vector $\left(\begin{array}{c}\dot{\lambda} \\ i\end{array}\right)$ of the branch of the real eigenvalue path at $\left(\lambda^{0}, t^{0}\right)$ is $\left(\begin{array}{c} \pm 1 \\ 0\end{array}\right)$. Since $\lambda^{0}$ is real, if $(\lambda, t) \in H^{-1}(0)$ is on the complex eigenvalue path passing through $\left(\lambda^{0}, t^{0}\right)$, then so is its conjugate $(\bar{\lambda}, t)$. Accordingly, at $\left(\lambda^{0}, t^{0}\right)$,

$$
\dot{\lambda}=\frac{d \lambda}{d s}=\lim _{\Delta s \rightarrow 0} \frac{\lambda-\bar{\lambda}}{\Delta s}
$$

which is purely imaginary: the tangent vector $\left(\begin{array}{l}\dot{\lambda} \\ i\end{array}\right)$ of the complex eigenvalue path at $\left(\lambda^{0}, t^{0}\right)$ must be $\left(\begin{array}{c} \pm i \\ 0\end{array}\right)$ (also see Figure 2 ).

We now proceed to prove Proposition 3.1.

Definition. Let $f(z)=a_{0}+a_{1} z+\cdots+a_{n} z^{n}$ and $g(z)=b_{0}+b_{1} z+\cdots+b_{m} z^{m}$ be polynomials of degree $n$ and $m$, respectively. The determinant of the $(n+m) \times(n+m)$ matrix

$$
\left(\begin{array}{cccccccc}
a_{0} & a_{1} & a_{2} & \cdots & \cdots & a_{n} & & \\
& \cdots & \cdots & \cdots & \cdots & \cdots & \cdots & \\
& & a_{0} & a_{1} & a_{2} & \cdots & \cdots & a_{n} \\
b_{0} & b_{1} & b_{2} & \cdots & \cdots & b_{m} & & \\
& \cdots & \cdots & \cdots & \cdots & \cdots & \cdots & \\
& & b_{0} & b_{1} & b_{2} & \cdots & \cdots & b_{m}
\end{array}\right)
$$

is called the resultant of $f$ and $g$. The resultant of a polynomial and its derivative is called the discriminant of the polynomial.

The following two lemmas can be found in [17].

Lemma 3.2. Two polynomials have a common nonconstant factor if and only if their resultant is zero.

Lemma 3.3. A polynomial has a multiple root if and only if its discriminant is zero.

The next lemma yields a criterion for an $n \times n$ irreducible upper Hessenberg matrix to have no multiple eigenvalues. 
Lemma 3.4. For $m$ real numbers $a_{1}, \ldots, a_{m}$, there exists a subset $C_{0} \subset \mathbf{R}$ consisting of at most $m-1$ elements such that the polynomial

$$
f(z)=a_{m} z^{m}+\cdots+a_{1} z+a_{0}
$$

has no multiple zeros if $a_{0} \notin C_{0}$.

Proof. The polynomial $f(z)$ has a multiple zero $z_{0}$ if and only if $f\left(z_{0}\right)=$ $f^{\prime}\left(z_{0}\right)=0$. Its derivative $f^{\prime}(z)$ is a polynomial of degree $m-1$ which is independent of $a_{0}$. Let $z_{1}, \ldots, z_{m-1}$ be the zeros of $f^{\prime}(z)$. For each $j=1, \ldots, m-1, z_{j}$ is a multiple zero of $f$ if and only if $a_{0}=$ $-\left(a_{m} z_{j}^{m}+\cdots+a_{1} z_{j}\right)$.

Corollary 3.5. Given an $m \times m$ irreducible upper Hessenberg matrix $B=\left(b_{i j}\right)$, there is an open subset $Q \subset \mathbf{R}$ which contains all $\mathbf{R}$ except for at most $m-1$ real numbers such that $B$ has no multiple eigenvalue if $b_{1 m} \in Q$.

Proof. Let $f(\lambda)=\operatorname{det}[B-\lambda I]$. A straightforward verification shows that the constant term of $f(\lambda)$ can be written as $K b_{1 m}+c$, where $K=\prod_{j=2}^{m} b_{j, j-1} \neq 0$ and the other terms of $f(\lambda)$ and $c$ are independent of $b_{1 m}$.

Proof of Proposition 3.1. (i) Both $D_{1}$ and $D_{2}$ are irreducible upper Hessenberg matrices. By Corollary 3.5 , there exist open subsets $\widetilde{Q}_{1}, \widetilde{Q}_{2} \subset \mathbf{R}$ with full measures such that neither $D_{1}$ nor $D_{2}$ has multiple eigenvalues if their entries $d_{1, p} \in \widetilde{Q}_{1}$ and $d_{p+1, n} \in \widetilde{Q}_{2}$. In addition, we want to show that there exists an open subset $\hat{Q}_{1} \subset \mathbf{R}^{2}$ with full measure such that if $\left(d_{1, p}, d_{p+1, n}\right) \in \widehat{Q}_{1}$, then $D_{1}$ and $D_{2}$ have no common eigenvalues. Let $d_{p+1, n} \equiv \alpha \in \widetilde{Q}_{2}$, and let $\lambda_{1}(\alpha), \ldots, \lambda_{n-p}(\alpha)$ be the eigenvalues of $D_{2}$ considered as functions of $\alpha$. Each $\lambda_{j}(\alpha)$ is a continuous function of $\alpha$. If $\lambda_{j}(\alpha), j \in\{1, \ldots, n-p\}$, is also an eigenvalue of $D_{1}$, then $d_{1, p}$ must make $\operatorname{det}\left[D_{1}-\lambda_{j}(\alpha) I\right]=0$, and it is clear that only one such value of $d_{1, p} \in \mathbf{C}$ exists for each $\lambda_{j}(\alpha)$ (see the proof of Corollary 3.5). That is, there are $n-p$ values $s_{1}(\alpha), \ldots, s_{n-p}(\alpha)$ (possibly repeated) in $\mathbf{C}$ for $d_{1, p}$ to be such that $D_{1}$ and $D_{2}$ have common eigenvalues. These $s_{j}$ 's are continuous functions of $\lambda_{j}$ (again, see the proof of Corollary 3.5). Thus, they are continuous functions of $\alpha$. Let $E$ be the closure of the set $\left\{\left(d_{1, p}, d_{p+1, n}\right) \mid d_{p+1, n} \in \widetilde{Q}_{2}, d_{1, p} \in\left\{s_{1}\left(d_{p+1, n}\right), \ldots, s_{n-p}\left(d_{p+1, n}\right)\right\}\right\} \cap \mathbf{R}^{2}$, which is obviously a subset of $\mathbf{R}^{2}$ with measure zero. Let $\widehat{Q}=\mathbf{R}^{2} \backslash E$. If $\left(d_{1, p}, d_{p+1, n}\right) \in \widehat{Q}$, then $D_{1}$ and $D_{2}$ have no common eigenvalues. Let $Q_{1}=$ $\left(\widetilde{Q}_{1} \times \widetilde{Q}_{2}\right) \cap \widehat{Q}$. For every $\left(d_{1, p}, d_{p+1, n}\right) \in Q_{1}$, the matrix $D$ has no multiple eigenvalues. $Q_{1}$ is clearly an open set with full measure.

(ii) Split $H, \lambda$, and $\mathbf{x}$ into their real and imaginary parts:

$$
\begin{aligned}
\lambda & =\xi+\eta i, \\
\mathbf{x} & =\mathbf{y}+\mathbf{z i}, \\
H(\xi+\eta i, t) & =F(\xi+\eta i, t)+G(\xi+\eta i, t) i
\end{aligned}
$$

Considering $\mathbf{C}$ as $\mathbf{R}^{2}$ and $d_{1, n-1}$ and $d_{1 n}$ as variables, we may rewrite equa- 
tion (3.1) as

$$
\begin{aligned}
& H\left(\xi+\eta i, t, d_{1, n-1}, d_{1 n}\right)=\left[\begin{array}{l}
F\left(\xi+\eta i, t, d_{1, n-1}, d_{1 n}\right) \\
G\left(\xi+\eta i, t, d_{1, n-1}, d_{1 n}\right)
\end{array}\right] \\
& =C(t)\left[\begin{array}{l}
\cdots+\left(t a_{1, n-1}+(1-t) d_{1, n-1}\right) y_{n-1}+\left(t a_{1, n}+(1-t) d_{1, n}\right) y_{n} \\
\cdots+\left(t a_{1, n-1}+(1-t) d_{1, n-1}\right) z_{n-1}+\left(t a_{1, n}+(1-t) d_{1, n}\right) z_{n}
\end{array}\right]
\end{aligned}
$$

where $C(t)=t \prod_{j=2}^{n} a_{j, j-1}$ and

$$
a_{n, n-1}(t)\left(y_{n-1}+z_{n-1} i\right)+\left(a_{n n}(t)-\xi-\eta i\right)\left(y_{n}+z_{n} i\right)=0
$$

with $y_{n}=1$ and $z_{n}=0$. Here, $a_{n, n-1}(t)=t a_{n, n-1}+(1-t) d_{n, n-1} \neq 0$. Thus,

$$
y_{n-1}=-\frac{\left(a_{n n}(t)-\xi\right)}{a_{n, n-1}(t)}, \quad z_{n-1}=\frac{\eta}{a_{n, n-1}(t)},
$$

and

$$
\begin{aligned}
\mathscr{D} H & =\left[\begin{array}{ccccc}
F_{\xi} & -G_{\xi} & F_{t} & F_{d_{1, n-1}} & F_{d_{1 n}} \\
G_{\xi} & F_{\xi} & G_{t} & G_{d_{1, n-1}} & G_{d_{1 n}}
\end{array}\right] \\
& =\left[\begin{array}{ccccc}
F_{\xi} & -G_{\xi} & F_{t} & F_{d_{1, n-1}} & C(t)(1-t) \\
G_{\xi} & F_{\xi} & G_{t} & \frac{C(t)(1-t) \eta}{a_{n, n-1}(t)} & 0
\end{array}\right] .
\end{aligned}
$$

Since $\eta \neq 0$, we have $\operatorname{rank}[\mathscr{D} H]=2$. From Sard's Theorem (see, e.g., $[1, \S 2]$ ), there exists a subset $Q_{2} \subset \mathbf{R}^{2}$ with full measure such that if $\left(d_{1, n-1}, d_{1 n}\right) \in Q_{2}$ then

$$
\mathscr{D} H=\left[H_{\lambda}, H_{t}\right]=\left(\begin{array}{ccc}
F_{\xi} & -G_{\xi} & F_{t} \\
G_{\xi} & F_{\xi} & G_{t}
\end{array}\right)
$$

is of full rank for all $(\lambda, t) \in H^{-1}(0) \cap[(\mathbf{C} \backslash \mathbf{R}) \times(0,1)]$.

(iii) Let $U=\mathbf{R} \times(0,1)$ and $V=\mathbf{R}$. Consider $d_{1 n}$ to be a variable of $H: U \times V \rightarrow \mathbf{R}$, and write

$$
H\left(\lambda, t, d_{1 n}\right)=C(t)\left[\left(a_{11}(t)-\lambda I\right) x_{1}+a_{12}(t) x_{2}+\cdots+\left(t a_{1 n}+(1-t) d_{1 n}\right) x_{n}\right] .
$$

With $x_{n}=1$, we have $\mathscr{D} H=\left[H_{\lambda}, H_{t}, C(t)(1-t)\right]$, which is of full rank. By Sard's Theorem, there exists a subset $Q_{3}$ in $\mathbf{R}$ with full measure such that if $d_{1 n} \in Q_{3}$ then $\mathscr{D} H=\left(H_{\lambda}, H_{t}\right)$ is of rank (real dimension) 1 for all $(\lambda, t) \in H^{-1}(0) \cap[\mathbf{R} \times(0,1)]$.

To satisfy (i), (ii), and (iii) simultaneously, we let

$$
Q=\left(Q_{1} \times \mathbf{R}^{2}\right) \cap\left(\mathbf{R}^{2} \times Q_{2}\right) \cap\left(\mathbf{R}^{3} \times Q_{3}\right) .
$$

Proposition 3.6. There exists an open set $Q_{0} \in \mathbf{R}^{2}$ with full measure such that if $\left(d_{1, n-1}, d_{1 n}\right) \in Q_{0}$, then for each $t \in(0,1)$, any eigenvalue $\lambda$ of $A(t)$ has multiplicity less than or equal to 2.

Proof. Consider $H=\operatorname{det}[A(t)-\lambda I]$ to be a polynomial in $\lambda$ with parameters $t, d_{1 n-1}$, and $d_{1 n}$. Let $f\left(t, d_{1, n-1}, d_{1 n}\right)$ and $g\left(t, d_{1, n-1}\right)$ be the discriminants of $H$ and $H_{\lambda}$, respectively, where $g$ is independent of $d_{1 n}$. Obviously, both $f$ and $g$ are not identically zero. Consider $f$ and $g$ to be polynomials in $t$ with parameters $d_{1, n-1}$ and $d_{1 n}$, and let $k\left(d_{1, n-1}, d_{1 n}\right)$ be the resultant of $f$ and $g$. Evidently, if $A(t)$ has a triple eigenvalue at some $t_{0}$ for fixed parameters $d_{1, n-1}^{*}$ and $d_{1 n}^{*}$, then $k\left(d_{1, n-1}^{*}, d_{1 n}^{*}\right)$ must be zero. It is easy to see that $k\left(d_{1, n-1}, d_{1 n}\right)$ is not identically zero. Indeed, for fixed $d_{1, n-1}$, there 
are only finitely many zeros $t_{1}, \ldots, t_{l}$ of $g$, and for this $d_{1, n-1}$ and each $t_{j}$, $j=1, \ldots, l$, there are only finitely many $d_{1 n}$ which make $f$ zero. Therefore, we can easily choose $d_{1, n-1}$ and $d_{1 n}$ such that $f$ and $g$ have no common zeros in $t$.

The zeros of $k\left(d_{1, n-1}, d_{1 n}\right)$ form a one-dimensional algebraic set in $\mathbf{R}^{2}$. We may choose $Q_{0}=\mathbf{R}^{2} \backslash k^{-1}(0)$, which is open and dense with full measure.

\section{Following the eigenvalue paths}

In the previous section, we showed that, theoretically, the desired regularity and simple bifurcation of the eigenvalue paths can be obtained with probability one by choosing four entries $\alpha=\left(d_{1 p}, d_{p+1, n}, d_{1, n-1}, d_{1 n}\right)$ of the initial matrix $D=\left(d_{i j}\right)$ at random. In practice, in order to apply the strategy of "divide and conquer," we let

$$
\begin{aligned}
& d_{i j}=a_{i j} \quad \text { if }(i, j) \neq(p+1, p), \\
& d_{p+1, p}=0 \quad \text { if }(i, j)=(p+1, p) \text {, }
\end{aligned}
$$

for a certain $p \approx n / 2$. We intend to perturb the parameters in $\boldsymbol{\alpha}$ when we encounter singularities, which, however, never occurred in our extensive numerical experiments. Apparently, the roundoff errors in solving for the eigenvalues of the matrix $D$ usually provide sufficient perturbations.

4.1. Following the real eigenvalue paths. Curve jumping is the most serious difficulty in following eigenvalue paths. Namely, when an eigenvalue path $\Gamma_{1}$ is followed, we may inadvertently jump to an eigenvalue path $\Gamma_{2}$ which passes close to $\Gamma_{1}$. Usually, this phenomenon only occurs in following real eigenvalue paths. When a complex eigenvalue path is followed, the path lies in $\mathbf{C} \times[0,1]$, which has one more dimension, and there is more room for maneuvering. Hence, when we trace a real eigenvalue path, special attention must be paid to prevent curve jumping. In the following, we first give several special features of our homotopy which are particularly useful in virtually eliminating the possibility of curve jumping occurring.

For the choice of the initial matrix $D$ in (4.1),

$$
\begin{aligned}
A(t) & =(1-t) D+t A \\
& =\left(\begin{array}{ccccccc}
a_{11} & \ldots & \ldots & \ldots & \ldots & \ldots & a_{1 n} \\
a_{21} & a_{22} & \ldots & \ldots & \ldots & \ldots & \vdots \\
& a_{32} & \ddots & & & & \vdots \\
& & \ddots & a_{p p} & \ldots & \ldots & \vdots \\
& & & t a_{p+1, p} & \ddots & & \vdots \\
& & & & \ddots & \ddots & \vdots \\
& & & & & a_{n, n-1} & a_{n n}
\end{array}\right) .
\end{aligned}
$$

Notice that $t$ only occurs in the $(p+1, p)$ entry. From simple observations, the homotopy $H(\lambda, t)=\operatorname{det}[A(t)-\lambda I]$ can be written as

$$
H(\lambda, t)=f_{1}(\lambda)+t f_{2}(\lambda),
$$

where $f_{1}$ and $f_{2}$ are polynomials in $\lambda$ of degrees $n$ and $n-2$, respectively. 


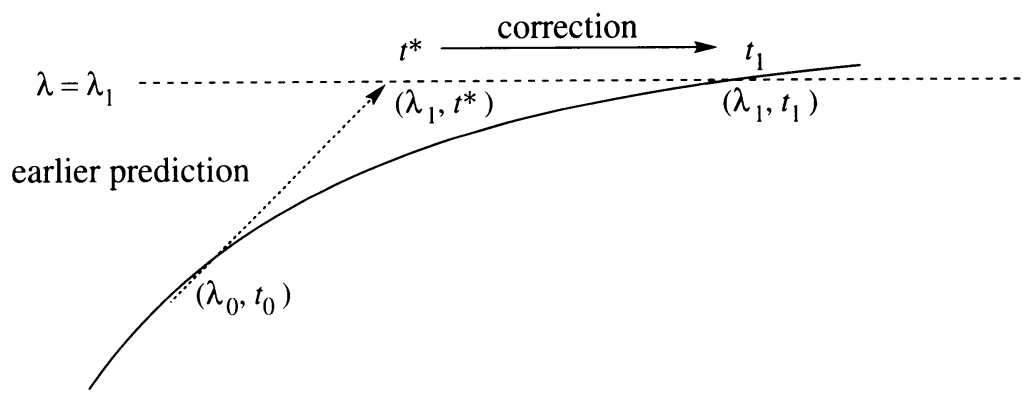

FIGURE 3. Newton iteration with fixed $\lambda=\lambda_{1}$

The representation of $H$ in (4.2) leads to the following very useful properties:

(i) Given any $\lambda_{0} \in \mathbf{R}$, if $f_{2}\left(\lambda_{0}\right) \neq 0$, there exists a unique $t_{0} \in \mathbf{R}$ such that $H\left(\lambda_{0}, t_{0}\right)=0$. Since $f_{2}$ has at most $n-2$ real zeros, any nonconstant component $\lambda(s)$ of any real eigenvalue path $(\lambda(s), t(s))$ must be monotone. Consequently, if

$$
\cdots<\lambda_{r-1}^{0}<\lambda_{r}^{0}<\lambda_{r+1}^{0}<\cdots
$$

are real eigenvalues of $D$, the eigenvalue path $(\lambda(s), t(s))$ emanating from $\left(\lambda_{r}^{0}, 0\right)$ will stay in $\left(\lambda_{r}^{0}, \lambda_{r+1}^{0}\right) \times(0,1)$ if $\lambda(s)$ is monotonically increasing, and in $\left(\lambda_{r-1}^{0}, \lambda_{r}^{0}\right) \times(0,1)$ if $\lambda(s)$ is monotonically decreasing. Based on this property, when a real eigenvalue path is followed, we may keep the eigenvalue path to stay within proper boundaries to prevent jumping.

(ii) Since $H(\lambda, t)$ is linear in $t$, for any $t^{*} \in(0,1)$ and any $\lambda_{1} \in \mathbf{R}$ with $f_{2}\left(\lambda_{1}\right) \neq 0$, the one-step Newton iteration gives

$$
t_{1}=t^{*}-\frac{H\left(\lambda_{1}, t^{*}\right)}{H_{t}\left(\lambda_{1}, t^{*}\right)}
$$

for which $H\left(\lambda_{1}, t_{1}\right)=0$. (See Figure 3.)

Another important property of real eigenpaths is the following degreepreserving property.

Proposition 4.1. Let

$$
P=\left\{(\lambda(s), t(s)) \in \mathbf{R} \times[0,1]: s \in\left[S_{0}, S_{1}\right]\right\}
$$

be a segment of an eigenpath which contains no bifurcation point. Then

$$
\operatorname{deg}[\lambda(s), t(s)] \equiv \operatorname{sign}\left[H_{\lambda}(\lambda(s), t(s))\right]=\text { const }
$$

for $s \in\left[S_{0}, S_{1}\right]$.

Proof. The function $H_{\lambda}$ is continuous and is equal to zero only at bifurcation points.

From this proposition, and property (i) above, when tracing a real eigenpath, we may check its degree and keep the eigenvalue path within the proper boundary to prevent jumping.

To follow a real eigenvalue path $\Gamma=(\lambda(s), t(s))$, let $\left(\lambda_{0}, t_{0}\right)$ be a point on $\Gamma$. We first calculate the tangent vector $(\dot{\lambda}, \dot{t})$ at $\left(\lambda_{0}, t_{0}\right)$. When arc length is 
used as the parameter of the eigenvalue path, the tangent vector can be obtained by solving the following system:

$$
\begin{aligned}
H_{\lambda} \dot{\lambda}+H_{t} \dot{t} & =0, \\
\dot{\lambda}^{2}+\dot{t}^{2} & =1 .
\end{aligned}
$$

As described in $\S 2$, both $H_{\lambda}$ and $H_{t}$ can be evaluated efficiently by Hyman's method. The sign of $\dot{t}$ is always chosen to be positive.

With the tangent vector $(\dot{\lambda}, \dot{t})$ at hand, we make the prediction $\left(\lambda_{1}^{0}, t_{1}^{0}\right)=$ $\left(\lambda_{0}, t_{0}\right)+\delta(\dot{\lambda}, \dot{t})$ with a step size $\delta>0$. This prediction will be the initial point of the correction iteration. The correction can be carried out in two ways:

1. If $t_{1}^{0} \neq 1$ and $|\dot{\lambda}|$ is not too small, say $|\dot{\lambda}|>10^{-4}$, then the slope $d \lambda / d t$ is not close to zero. That is, locally the eigenvalue path is not close to horizontal. Hence, the horizontal line $\lambda=\lambda_{1}^{0}$ should intersect the eigenvalue path. In this case, we may perform the correction on the horizontal line $\lambda=\lambda_{1}^{0}$. Namely, we use what we described in formula (4.3):

$$
\begin{aligned}
& \lambda_{1}=\lambda_{1}^{0}, \\
& t_{1}=t_{1}^{0}-\frac{H\left(\lambda_{1}^{0}, t_{1}^{0}\right)}{H_{t}\left(\lambda_{1}^{0}, t_{1}^{0}\right)} .
\end{aligned}
$$

The advantage of this iteration is that only one step of Newton's iteration is necessary to obtain $t_{1}$ from $t_{1}^{0}$ for fixed $\lambda_{1}^{0}$ for which $H\left(\lambda_{1}, t_{1}\right)=0$.

2. If $|\dot{\lambda}| \approx 0$, the horizontal correction formula in method 1 is not applicable, since the horizontal line $\lambda=\lambda_{1}^{0}$ may not intersect the eigenvalue path which is nearly horizontal. In this case, however, the vertical line $t=t_{1}^{0}$ will intersect the eigenvalue path. Thus, we can correct $\lambda_{1}^{0}$ for fixed $t=t_{1}^{0}$ by using the formula

$$
\lambda_{1}^{m+1}=\lambda_{1}^{m}-\frac{H\left(\lambda_{1}^{m}, t_{1}^{0}\right)}{H_{\lambda}\left(\lambda_{1}^{m}, t_{1}^{0}\right)}, \quad m=0,1, \ldots
$$

We end the iteration if $\left|H\left(\lambda_{1}^{m}, t_{1}^{0}\right) / H_{\lambda}\left(\lambda_{1}^{m}, t_{1}^{0}\right)\right|<\varepsilon$, where $\varepsilon$ is a preset error tolerance, and let $\left(\lambda_{1}, t_{1}\right)=\left(\lambda_{1}^{m+1}, t_{1}^{0}\right)$.

After obtaining $\left(\lambda_{1}, t_{1}\right)$, we must check

(a) if $\left(\lambda_{1}, t_{1}\right)$ is in the proper region described in property $(\mathrm{i})$;

(b) if $\operatorname{sign}\left[H_{\lambda}\left(\lambda_{0}, t_{0}\right)\right]=\operatorname{sign}\left[H_{\lambda}\left(\lambda_{1}, t_{1}\right)\right]$.

In $(\mathrm{a})$, if $\left(\lambda_{1}, t_{1}\right)$ is out of the region, path jumping occurs. We thus discard $\left(\lambda_{1}, t_{1}\right)$, repeat the prediction with half the step size, and correct again. For (b), if the sign of $H_{\lambda}$ changes at $\left(\lambda_{1}, t_{1}\right)$, then either path jumping or bifurcation occurs. In this case, we shall try the bifurcation treatment first (see $\S 4.3$ ). If there is no bifurcation between $\left(\lambda_{0}, t_{0}\right)$ and $\left(\lambda_{1}, t_{1}\right)$, then we conclude that curve jumping has taken place. We cut the step size in half and repeat the prediction and correction at $\left(\lambda_{0}, t_{0}\right)$.

If $\left(\lambda_{1}, t_{1}\right)$ passes both tests (a) and (b), i.e., $\left(\lambda_{1}, t_{1}\right)$ lies in the proper region and no sign change for $H_{\lambda}$ occurs at $\left(\lambda_{1}, t_{1}\right)$, we accept $\left(\lambda_{1}, t_{1}\right)$ as a new point on the eigenvalue path $\Gamma$.

4.2. Following a complex eigenvalue path. Complex eigenvalue paths appear in pairs. If $(\lambda(s), t(s))$ is a complex eigenvalue path, so is its conjugate $(\bar{\lambda}(s)$, $t(s))$. We need to follow only one of them, say, the one with positive imaginary 
part in $\lambda(s)$. Let $\left(\lambda_{0}, t_{0}\right)$ be a point on $\Gamma=(\lambda(s), t(s))$ with $\operatorname{Im}(\lambda(s))>0$. The tangent vector $(\dot{\lambda}, \dot{t})$ at the point $\left(\lambda_{0}, t_{0}\right)$ is the solution of the following system of equations:

$$
\begin{aligned}
H_{\lambda} \dot{\lambda}+H_{t} \dot{i} & =0, \\
\dot{\bar{\lambda}} \dot{\lambda}+\dot{t}^{2} & =1 \quad(\dot{i}>0) .
\end{aligned}
$$

After finding the tangent vector $(\dot{\lambda}, \dot{t})$, we make the prediction

$$
\left(\lambda_{1}^{0}, t_{1}^{0}\right)=\left(\lambda_{0}, t_{0}\right)+\delta(\dot{\lambda}, \dot{t})
$$

with step size $\delta>0$. Since $H(\lambda, t)=0$ is a system of two real equations in three variables (counting real and imaginary parts of $\lambda$ ), in order to apply Newton's method for the correction, we add one more equation. This equation is in the form of a plane

$$
\operatorname{Re}\left[\bar{u}\left(\lambda-\lambda_{1}^{0}\right)\right]+v\left(t-t_{1}^{0}\right)=0,
$$

where $(u, v) \in \mathbf{C} \times \mathbf{R}$. There are three options for the choice of the plane in (4.4):

(i) When $\dot{t} \approx 1$, we choose $(u, v)=(0,1)$. With this choice, the correction is executed for fixed $t=t_{1}^{0}$. The Newton iteration has the simple form

$$
\lambda_{1}^{m+1}=\lambda_{1}^{m}-\frac{H\left(\lambda_{1}^{m}, t_{1}^{0}\right)}{H_{\lambda}\left(\lambda_{1}^{m}, t_{1}^{0}\right)}
$$

and the cost is as low as $4 n^{2}+O(n)$ floating-point operations per step.

(ii) If $\dot{t} \approx 0$, we choose $(u, v)=(\dot{\lambda}, \dot{t})$. The correction in this case is in the plane perpendicular to the tangent vector.

(iii) When bifurcation is suspected, i.e., $\operatorname{Im}\left(\lambda_{1}^{0}\right)>0$, we choose $(u, v)=$ $(i, 0)$ because $(i, 0)$ is the tangent vector at the bifurcation point. This case will be discussed in more detail when we treat bifurcation in the next subsection.

We then perform Newton's iteration on the equation $H(\lambda, t)=0$ augmented by (4.4) with proper $(u, v)$, starting from the initial point $\left(\lambda_{1}^{0}, t_{1}^{0}\right)$. If the iteration does not converge, we cut the step size in half and repeat the predictioncorrection step again at $\left(\lambda_{0}, t_{0}\right)$. If Newton's iteration converges, let $\left(\lambda_{1}, t_{1}\right)$ be the limit point. If $\operatorname{Im}\left(\lambda_{1}\right)>0$, then there is no bifurcation between $\left(\lambda_{0}, t_{0}\right)$ and $\left(\lambda_{1}, t_{1}\right)$, and we thus accept $\left(\lambda_{1}, t_{1}\right)$ as a new point on $\Gamma$. If $\operatorname{Im}\left(\lambda_{1}\right)<0$, then there is bifurcation between $\left(\lambda_{0}, t_{0}\right)$ and $\left(\lambda_{1}, t_{1}\right)$. We then follow the bifurcation treatment described in the following subsection.

4.3. Bifurcation. Bifurcation cannot be avoided in our homotopy. Therefore, we must develop an efficient algorithm to identify the bifurcation point and continuously follow the bifurcation branches. In [13], a method is introduced to detect and pass the bifurcation point for a real homotopy. However, the simplicity of our homotopy and associated eigenvalue paths makes room for a much more efficient method.

4.3.1. Real-to-complex bifurcation. As we mentioned before, if $\left(\lambda_{0}, t_{0}\right)$ and $\left(\lambda_{1}, t_{1}\right)$ are two consecutive points obtained on a real eigenvalue path $\Gamma=$ $(\lambda(s), t(s))$, and the sign of $H_{\lambda}$ at $\left(\lambda_{1}, t_{1}\right)$ differs from the sign of $H_{\lambda}$ at $\left(\lambda_{0}, t_{0}\right)$, then a bifurcation point $\left(\lambda^{0}, t^{0}\right)$ exists between these two points, at 


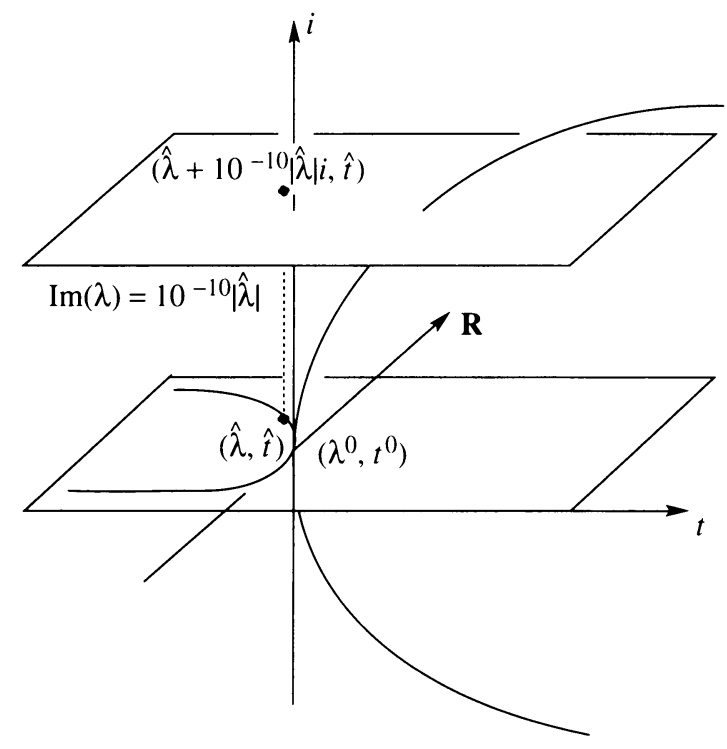

FIGURE 4. Space lifting in bifurcation situation

which $H_{\lambda}$ must vanish. We first use linear interpolation to approximate $\lambda^{0}$; that is, we let

Then, we fix $\hat{\lambda}$ and solve for $\hat{t}$ by

$$
\hat{\lambda}=\lambda_{0}-H_{\lambda}\left(\lambda_{0}, t_{0}\right)=\frac{\lambda_{0}-\lambda_{1}}{H_{\lambda}\left(\lambda_{0}, t_{0}\right)-H_{\lambda}\left(\lambda_{1}, t_{1}\right)} .
$$

$$
\hat{t}=t_{0}-\frac{H\left(\hat{\lambda}, t_{0}\right)}{H_{t}\left(\hat{\lambda}, t_{0}\right)} .
$$

The point $(\hat{\lambda}, \hat{t})$ will be taken as an approximation of the bifurcation point $\left(\lambda^{0}, t^{0}\right)$. With the "lifting" technique described below, the accuracy of the approximation $(\hat{\lambda}, \hat{t})$ becomes much less important.

From $(\hat{\lambda}, \hat{t})$, we make a prediction of the complex bifurcation branch by lifting $(\hat{\lambda}, \hat{t})$ into the complex space $\mathbf{C} \times[0,1]$. That is, we add $10^{-10}|\hat{\lambda}| i$ to $\hat{\lambda}$ and take $\left(\hat{\lambda}+10^{-10}|\hat{\lambda}| i, \hat{t}\right)$ as our prediction. Then the correction is carried out on the plane $\operatorname{Im}(\lambda)=10^{-10}|\hat{\lambda}|$ (option (iii) of the plane in (4.4)) (see Figure 4). This procedure is very efficient. The real-to-complex space transition can be completed without computing the tangent vector at $(\hat{\lambda}, \hat{t})$.

Remark. Curve jumping can also cause a sign change of $H_{\lambda}$ at $\left(\lambda_{1}, t_{1}\right)$, since two real eigenvalue paths next to each other have different degrees, i.e., +1 and -1 , among them. This situation can easily be detected. If $\hat{t}$ in (4.5) is outside the interval $[0,1]$, or the correction after "lifting" does not converge, then there is no bifurcation between $\left(\lambda_{0}, t_{0}\right)$ and $\left(\lambda_{1}, t_{1}\right)$. Evidently, $\left(\lambda_{1}, t_{1}\right)$ is on the wrong eigenvalue path and must be discarded. We then cut the step size and repeat the prediction-correction step at $\left(\lambda_{0}, t_{0}\right)$.

4.3.2. Complex-to-real bifurcation. Let $\left(\lambda_{0}, t_{0}\right)$ and $\left(\lambda_{1}, t_{1}\right)$ be two consecutive points obtained on a complex eigenvalue path. If $\operatorname{Im}\left(\lambda_{0}\right)>0$ and $\operatorname{Im}\left(\lambda_{1}\right)<$ 0 , then a bifurcation point $\left(\lambda^{0}, t^{0}\right)$ with $\operatorname{Im}\left(\lambda^{0}\right)=0$ exists. We proceed by 
basically reversing the steps in real-to-complex bifurcation. To approximate $\left(\lambda^{0}, t^{0}\right)$, we let $\delta_{*}$ be the solution of

$$
\operatorname{Im}\left(\lambda_{0}+\delta \dot{\lambda}\right)=10^{-10}\left|\lambda_{0}\right|
$$

where $(\dot{\lambda}, \dot{t})$ is the tangent vector at $\left(\lambda_{0}, t_{0}\right)$. Making a new prediction at $\left(\lambda_{0}, t_{0}\right)$ with tangent vector $(\dot{\lambda}, \dot{t})$ at $\left(\lambda_{0}, t_{0}\right)$ and step size $\delta_{*}$, and carrying out the correction on the plane $\operatorname{Im}(\lambda)=10^{-10}\left|\lambda_{0}\right|$ (i.e., option (iii) of the plane in (4.4)), yields an approximation $(\hat{\lambda}, \hat{t})$ of the bifurcation point $\left(\lambda^{0}, t^{0}\right)$. We then project $(\hat{\lambda}, \hat{t})$ from $\mathbf{C} \times[0,1]$ into $\mathbf{R} \times[0,1]$ by taking $\left(\operatorname{Re}\left(\hat{\lambda}_{0}\right), \hat{t}\right)$ as a prediction of the real bifurcation branch. The correction is made on the line $\lambda=\operatorname{Re}(\hat{\lambda})$ to obtain a point $(\hat{\lambda}, t)$ on a real bifurcation branch. From our bifurcation analysis, there are two real bifurcation branches with tangent vector $(1,0)$ and $(-1,0)$, respectively. We thus perform prediction-correction with each tangent vector separately to trace both eigenvalue paths.

4.4. Step size control. (i) Initial step size. When following an eigenvalue path of

$$
H(\lambda, t)=\operatorname{det}([(1-t) D+t A]-\lambda I)=0
$$

at an initial point $\left(\lambda_{0}, 0\right)$, where $\lambda_{0}$ is an eigenvalue of $D$, our first attempt is to choose the initial step size $\delta=1$. The point $\left(\lambda_{0}, 1\right)$ will then be taken as a prediction point, which is followed by Newton's correction at $t=1$. This procedure, 0 -order prediction with step size 1 followed by Newton's correction, is the same as applying Newton's iteration directly for solving the equation

$$
\operatorname{det}(A-\lambda I)=0
$$

by using $\lambda_{0}$ as a starting point. The choice of the initial matrix $D$ in (2) makes the eigenvalues of $D$ very close to the eigenvalues of $A$. Hence, Newton's iteration on $\operatorname{det}(A-\lambda I)=0$ with starting point $\lambda_{0}$ has great potential to converge. Indeed, our numerical results indicate that the vast majority (usually more than $90 \%$ ) of the eigenvalues of $A$ can be obtained in this way. If Newton's correction fails to converge, then we choose the step size in a standard way described below. In some sense, our homotopy continuation algorithm here mainly plays a backup role of directly applying Newton's iteration for solving $\operatorname{det}(A-\lambda I)=0$, starting from the eigenvalue of $D$.

When the above procedure with $\delta=1$ fails, we evaluate the tangent vector $\left(\dot{\lambda}_{0}, \dot{t}_{0}\right)$ at $\left(\lambda_{0}, 0\right)$. If $\dot{t}_{0}$ is close to 1 , then $\left|\dot{\lambda}_{0}\right| \approx 0$, since $\left|\dot{\lambda}_{0}\right|^{2}+\dot{t}_{0}^{2}=1$. So, the eigenvalue path is close to a straight line at $\left(\lambda_{0}, 0\right)$ and can tolerate large step size; we take $\delta=1 / \dot{t}_{0}$ in this case. When $\dot{t}_{0} \ll 1$, we let $\delta=\max \left\{0.01,|\dot{t}|^{5}\right\}$.

(ii) Cutting the step size. From a given point $\left(\lambda_{0}, t_{0}\right) \in H^{-1}(0)$ on an eigenvalue path, we make a prediction $\left(\lambda_{1}^{0}, t_{1}^{0}\right)=\left(\lambda_{0}, t_{0}\right)+\delta(\dot{\lambda}, \dot{t})$ with step size $\delta$. In the correction process, if the iteration does not show a "tendency to converge," we will repeat the prediction at $\left(\lambda_{0}, t_{0}\right)$ with step size $\delta / 2$. As criterion for judging the "tendency to converge" we use

$$
\left|\lambda_{1}^{k+1}-\lambda_{1}^{k}\right| \leq \frac{1}{5}\left|\lambda_{1}^{k}-\lambda_{1}^{k-1}\right| \text {. }
$$

(iii) Increasing the step size. When the prediction-correction step at $\left(\lambda_{0}, t_{0}\right)$ is successful, we obtain a new point $\left(\lambda_{1}, t_{1}\right)$ on the eigenvalue path. If the angle between the tangent vectors at these two points is small (say, less than $15^{\circ}$ ), 
then it appears that the eigenvalue path is quite flat and we double the step size in our first prediction attempt at $\left(\lambda_{1}, t_{1}\right)$. Otherwise, the last successful step size in achieving $\left(\lambda_{1}, t_{1}\right)$ is used for the prediction at $\left(\lambda_{1}, t_{1}\right)$.

(iv) Adjusting the step size. If the prediction $\left(\lambda_{0}, t_{0}\right)+\delta(\dot{\lambda}, \dot{t})$ gives $t_{0}+\delta \dot{t}>$ 1 , then we let $\delta=\left(1-t_{0}\right) / \dot{t}$, making the prediction reach the plane $t=1$.

\section{NUMERICAL RESULTS}

The eigenvalues of the initial matrix

$$
D=\left(\begin{array}{cc}
D_{1} & * \\
0 & D_{2}
\end{array}\right)
$$

consist of the eigenvalues of the submatrices $D_{1}$ and $D_{2}$, both irreducible upper Hessenberg matrices. The strategy of "divide and conquer" can certainly be repeated in finding the eigenvalues of $D_{1}$ and $D_{2}$, etc. However, our experience shows that the QR algorithm implemented in EISPACK [16], the HQR subroutine, is normally faster than our algorithm for $n \leq 25$. Therefore, we stop the "divide" procedure when the sizes of the submatrices are less than 25. We determine the eigenvalues of the submatrices by the $\mathrm{QR}$ algorithm and then start the "conquer" procedure consecutively.

We first tested our algorithm on $n \times n$ upper Hessenberg matrices $A=$ $\left(a_{i j}\right)$ with entries $-1 \leq a_{i j} \leq 1$ generated by a random number generator. The computations were done on a SPARC Station 1 in double precision. In comparing with $\mathrm{HQR}$ on a common basis of accuracy, we require the computed eigenvalues $\lambda_{1}, \ldots, \lambda_{n}$ to satisfy

$$
\left|\frac{1}{n} \sum_{j=1}^{n}\left(\lambda_{j}-a_{j j}\right)\right|<10^{-16},
$$

the same trace-accuracy HQR achieves.

For fixed matrix size $n$, we executed our algorithm on more than 20 different matrices that are consecutive in a preset random number sequence. The results are shown in Table 1. The efficiency of our algorithm is closely related to the amount of bifurcations one encounters in following the eigenvalue paths. Thus, for fixed $n$, the CPU time of each individual case varies in a relatively wide range. Nevertheless, the average CPU time is still quite encouraging. For comparison, the results for $\mathrm{HQR}$ on the same matrices are also listed in Table 1. While the potential of our method lies in its natural parallelism in the sense that each eigenvalue path can be followed independently, it is remarkable that our algorithm is strongly competitive on these examples even on serial computers. The parallel implementation of our algorithm using $n$ processors should increase the efficiency by a full power of $n$, making it an excellent candidate for advanced computer architectures. In contrast, the $\mathrm{QR}$ iteration is inherently highly serial.

In addition to testing the trace-accuracy given in (5.1), we also tested the difference between the corresponding eigenvalues evaluated by both methods. Let $\hat{\lambda}_{i}$ and $\tilde{\lambda}_{i}, i=1, \ldots, n$, be eigenvalues obtained by using HQR and our homotopy method, respectively. Put $\hat{\lambda}_{i}$ and $\tilde{\lambda}_{i}, i=1, \ldots, n$, in "ascending" 
TABLE 1. Time comparison between HQR and H-D

(H-D = Homotopy-Determinant method)

\begin{tabular}{|c|c|c|c|c|c|}
\hline \multirow{2}{*}{$\begin{array}{l}\text { Matrix } \\
\text { order }\end{array}$} & & \multicolumn{3}{|c|}{ time (seconds) } & \multirow{2}{*}{$\begin{array}{c}\text { Average time ratio } \\
\mathrm{HQR} / \mathrm{H}-\mathrm{D}\end{array}$} \\
\hline & & minimum & maximum & average & \\
\hline \multirow[t]{2}{*}{20} & HQR & $\overline{0.34}$ & 0.49 & 0.3973 & \multirow[b]{2}{*}{0.708} \\
\hline & H-D & 0.21 & 1.51 & 0.5609 & \\
\hline \multirow[t]{2}{*}{$\overline{25}$} & $\overline{\mathrm{HQR}}$ & $\overline{0.63}$ & "0.87 & 0.7388 & \multirow[b]{2}{*}{1.034} \\
\hline & H-D & 0.38 & 1.58 & 0.7144 & \\
\hline \multirow[t]{2}{*}{50} & HQR & 4.58 & 5.68 & 5.11 & \multirow[b]{2}{*}{1.29} \\
\hline & H-D & 2.01 & 7.72 & 3.96 & \\
\hline \multirow[t]{2}{*}{100} & HQR & 34.05 & $\overline{39.02}$ & 36.41 & \multirow[b]{2}{*}{1.59} \\
\hline & H-D & 11.99 & 40.72 & 22.90 & \\
\hline \multirow[t]{2}{*}{200} & $\overline{\mathrm{HQR}}$ & 254.52 & 281.57 & 266.30 & \multirow[b]{2}{*}{2.26} \\
\hline & H-D & 72.28 & 340.24 & 142.01 & \\
\hline \multirow[t]{2}{*}{300} & HQR & 643.18 & 660.50 & 651.84 & \multirow[b]{2}{*}{2.03} \\
\hline & H-D & 208.26 & 579.51 & 320.88 & \\
\hline \multirow[t]{2}{*}{400} & $\overline{\mathrm{HQR}}$ & 1536.61 & 1563.24 & 1549.40 & \multirow[b]{2}{*}{2.25} \\
\hline & H-D & 510.07 & 1779.93 & 689.47 & \\
\hline
\end{tabular}

order

$$
\begin{aligned}
& \hat{\lambda}_{1} \prec \hat{\lambda}_{2} \prec \cdots \prec \hat{\lambda}_{n}, \\
& \tilde{\lambda}_{1} \prec \tilde{\lambda}_{2} \prec \cdots \prec \tilde{\lambda}_{n},
\end{aligned}
$$

where $a \prec b$ means either (i) $\operatorname{Re}(a)<\operatorname{Re}(b)$ or (ii) $\operatorname{Re}(a)=\operatorname{Re}(b)$ but $\operatorname{Im}(a) \leq \operatorname{Im}(b)$. Then we found that the estimate

$$
\frac{\max _{1 \leq j \leq n}\left|\hat{\lambda}_{j}-\tilde{\lambda}_{j}\right|}{\|A\|}<1.0 \times 10^{-10}
$$

held on all of our testing examples. Apparently, both methods have about the same accuracy on random matrices.

As we discussed in the last section, 0-order prediction with step size one at an initial point $\left(\lambda_{0}, 0\right)$, where $\lambda_{0}$ is an eigenvalue of the initial matrix $D$, followed by Newton's correction at $t=1$, or equivalently, applying Newton's iteration directly to $\operatorname{det}(A-\lambda I)=0$ with starting point $\lambda_{0}$, has great potential to converge. If it converges, the corresponding eigenvalue of $A$ is obtained with one step in following the eigenvalue path. We shall call such an eigenvalue path an "easy path." We show in Table 2 (see next page) the percentage of the "easy paths" we found in each category. It can be seen that the overwhelming majority of the eigenvalue paths are "easy paths." Bifurcations are inevitable when real homotopies are used. We also show in Table 2 the bifurcation frequency, that is, the average number of bifurcation points encountered in following all eigenvalue paths for each matrix. It appears that the choice of the special form of the initial matrix $D$ and using the strategy of "divide and conquer" minimize the occurrence of the bifurcation points. In all our computations, curve jumping was never a problem before the final results were reached, and was effectively prevented by adjusting the step size.

Usually, eigenvalues of random matrices are well-conditioned. To test the accuracy of our algorithm on ill-conditioned eigenvalues, we constructed testing matrices with some ill-conditioned eigenvalues in the following way. 
TABLE 2. Rates of "easy" paths and bifurcations

\begin{tabular}{|c||c|c|}
\hline $\begin{array}{c}\text { Matrix order } \\
n\end{array}$ & $\begin{array}{c}\text { Percentage } \\
\text { of } \\
\text { "easy" paths }\end{array}$ & $\begin{array}{c}\text { Bifurcation frequency } \\
\text { (total bifurcation points on } \\
\text { all paths per matrix) }\end{array}$ \\
\hline 25 & $87.1 \%$ & 0.92 \\
\hline 50 & $92.3 \%$ & 1.27 \\
\hline 100 & $93.4 \%$ & 2.08 \\
\hline 200 & $95.7 \%$ & 2.78 \\
\hline 300 & $98.1 \%$ & 0.92 \\
\hline 400 & $96.0 \%$ & 1.56 \\
\hline
\end{tabular}

Let $A$ be a matrix in the block form

$$
A=\left(\begin{array}{ccc}
J & 0 & 0 \\
0 & J & 0 \\
0 & 0 & \widehat{A}
\end{array}\right),
$$

where $J$ is a $5 \times 5$ Jordan block with multiple eigenvalue 0 and $\widehat{A}$ is a $90 \times 90$ matrix of the following form:

$$
\widehat{A}=\left(\begin{array}{cccccccccc}
\times & \times & * & * & * & * & \ldots & \ldots & * & * \\
\times & \times & * & * & * & * & \cdots & & * & * \\
0 & 0 & \times & \times & * & * & & & * & * \\
0 & 0 & \times & \times & * & * & \ldots & \ldots & * & * \\
0 & 0 & 0 & 0 & \times & \times & & & * & * \\
0 & 0 & 0 & 0 & \times & \times & \ldots & \ldots & * & * \\
\vdots & & & & \ddots & \ddots & & & \vdots \\
\vdots & & & & & \ddots & \ddots & & \vdots \\
0 & 0 & & & & & & & \\
0 & 0 & \cdots & & \ldots & \ldots & 0 & \times & \times \\
& & & & & 0 & \times & \times
\end{array}\right),
$$

where the diagonal blocks consist of $2 \times 2$ matrices $\left(\begin{array}{ll}x & x \\ x & x\end{array}\right)$ with known and evenly distributed eigenvalues, and the $*$ 's above the diagonal blocks are randomly generated numbers. Thus, the matrix $A$ is a $100 \times 100$ matrix with a 10 -fold ill-conditioned eigenvalue 0 and 90 well-conditioned simple eigenvalues. Now we use a randomly chosen orthogonal dense matrix $U$ to make $U^{\top} A U$ a dense matrix. Then a standard algorithm is applied to reduce it to upper Hessenberg form. Let the resulting upper Hessenberg matrix be $B$ on which we perform both $\mathrm{HQR}$ and the homotopy method. The results for the ten ill-conditioned eigenvalues are listed in Table 3.

For the 90 known well-conditioned eigenvalues

$$
\Lambda_{1} \prec \Lambda_{2} \prec \cdots \prec \Lambda_{90}
$$

of $A$, let

$$
\hat{\lambda}_{1} \prec \hat{\lambda}_{2} \prec \cdots \prec \hat{\lambda}_{90} \quad \text { and } \quad \tilde{\lambda}_{1} \prec \tilde{\lambda}_{2} \prec \cdots \prec \tilde{\lambda}_{90}
$$

be eigenvalues obtained by HQR and the homotopy method, respectively. The respective accuracies are shown in Table 4 . These results clearly show that our method achieves about the same accuracy as HQR . 
TABLE 3. Results for ill-conditioned eigenvalues

\begin{tabular}{|c||c|c|}
\hline $\begin{array}{c}\text { exact } \\
\text { eigenvalue }\end{array}$ & $\begin{array}{c}\text { corresponding HQR } \\
\text { results }\end{array}$ & $\begin{array}{c}\text { corresponding homotopy } \\
\text { results }\end{array}$ \\
\hline 0 & -0.000880 & -0.000903 \\
\hline 0 & $-0.000730 \pm i 0.000530$ & $-0.000694 \pm i 0.000506$ \\
\hline 0 & $-0.000272 \pm i 0.000837$ & $-0.000276 \pm i 0.000852$ \\
\hline 0 & $0.000278 \pm i 0.000859$ & $0.000273 \pm i 0.000841$ \\
\hline 0 & $0.000712 \pm i 0.000517$ & $0.000727 \pm i 0.000529$ \\
\hline 0 & 0.000803 & 0.000817 \\
\hline
\end{tabular}

TABLE 4. Results for well-conditioned eigenvalues

\begin{tabular}{|l||l|}
\hline $\max _{1 \leq j \leq 90}\left|\hat{\lambda}_{j}-\Lambda_{j}\right|$ & $9.5300 \times 10^{-11}$ \\
\hline $\max _{1 \leq j \leq 90}\left|\tilde{\lambda}_{j}-\Lambda_{j}\right|$ & $7.6840 \times 10^{-11}$ \\
\hline $\max _{1 \leq j \leq 90}\left|\hat{\lambda}_{j}-\tilde{\lambda}_{j}\right|$ & $1.8460 \times 10^{-11}$ \\
\hline
\end{tabular}

\section{BIBLIOGRAPHY}

1. S-N. Chow, J. Mallet-Paret, and J. A. Yorke, Finding zeros of maps: homotopy methods that are constructive with probability one, Math. Comp. 32 (1978), 887-899.

2. M. T. Chu, A note on the homotopy method for linear algebraic eigenvalue problems, Linear Algebra Appl. 105 (1988), 225-236.

3. M. T. Chu, T. Y. Li, and T. Sauer, Homotopy method for general $\lambda$-matrix problems, SIAM J. Matrix Anal. Appl. 9 (1988), 528-536.

4. J. J. J. M. Cuppen, A divide and conquer method for the symmetric tridiagonal eigenproblem, Numer. Math. 36 (1981), 177-195.

5. J. J. Dongarra and M. Sidani, A parallel algorithm for the non-symmetric eigenvalue problem, Technical Report, Comp. Sci. Dept. CS-91-137, Univ. of Tennessee.

6. J. J. Dongarra and D. C. Sorensen, A fully parallel algorithm for the symmetric eigenvalue problem, SIAM J. Sci. Statist. Comput. 8 (1987), 139-154.

7. M. Hyman, Eigenvalues and eigenvectors of general matrices, presented at the 12th National Meeting of the Association for Computing Machinery, Houston, Texas, June 1957.

8. I. Ilse and E. Jessup, Solving the symmetric tridiagonal eigenvalue problem on the hypercube, SIAM J. Sci. Statist. Comput. 11 (1990), 203-229.

9. E. Jessup, Parallel solution of the symmetric eigenvalue problem, Ph.D. Thesis, Yale University, 1989.

10. T. Y. Li and N. H. Rhee, Homotopy algorithm for symmetric eigenvalue problems, Numer. Math. 55 (1989), 265-280.

11. T. Y. Li and T. Sauer, Homotopy method for generalized eigenvalue problems $A \mathbf{x}=\lambda B \mathbf{x}$, Linear Algebra Appl. 91 (1987), 65-74.

12. T. Y. Li, T. Sauer, and J. Yorke, Numerical solution of a class of deficient polynomial systems, SIAM J. Numer. Anal. 24 (1987), 435-451.

13. T. Y. Li, Z. Zeng, and L. Cong, Solving eigenvalue problems of real nonsymmetric matrices with real homotopies, SIAM J. Numer. Anal. 29 (1992), 229-248.

14. T. Y. Li, H. Zhang, and X. H. Sun, Parallel homotopy algorithm for symmetric tridiagonal eigenvalue problems, SIAM J. Sci. Statist. Comput. 12 (1991), 464-485.

15. S. S. Lo, B. Philippe, and A. Sameh, A multiprocessor algorithm for the symmetric tridiagonal eigenvalue problem, SIAM J. Sci. Statist. Comput. 8 (1987), 155-165. 
16. B. T. Smith et al., Matrix eigensystem routines-EISPACK guide, 2nd ed., Springer-Verlag, 1976.

17. B. L. Van der Waerden, Modern algebra. Vol. 1, Ungar, New York, 1949.

18. J. H. Wilkinson, Error analysis of floating-point computation, Numer. Math. 2 (1960), 319340 .

Department of Mathematics, Michigan State University, East Lansing, Michigan 48824-1027

E-mail address: li@mth.msu.edu

E-mail address: zeng@mth.msu.edu 